\title{
Pressure calculation for flows with moving surface boundaries from particle tracking velocimetry (PTV)
}

\author{
Reza Azadi and David S. Nobes* \\ Applied Thermofluids Lab., Department of Mechanical Engineering \\ University of Alberta, Edmonton, Canada \\ *david.nobes@ualberta.ca
}

The examples of flow conditions, where an object of a fixed or deformable body moves in a fluid, or the interface between the flow phases instantaneously changes its topology, are numerous in industry and natural sciences. The advent of particle image velocimetry (PIV) [1] and particle tracking velocimetry (PTV) [2] enabled the measurement of the instantaneous velocity fields in these types of complicated flow fields. As a next step, several methodologies have been developed in the past decade to calculate the pressure fields from PIV or PTV data [3,4]. These methods were developed based on the assumption of a stationary flow domain, with surface boundaries that are fixed and independent of time. This makes the current pressure calculation methods inapplicable to a flow domain with deformable moving surface boundaries. Also, for most of the two-phase flows, the capillary forces are significant and the pressure drop over the two-phase interface must be considered. Therefore, the current pressure calculators require an improvement in the formulation of the algorithms to account for the deformable volume conditions and the effect of the surface tension force. For the calculation of pressure from sparse PTV velocity data, firstly, a tessellation method is required to interconnect the irregularly spaced vectors in the flow field using a highquality mesh grid. The mesh must be dynamic and adjust itself to the moving boundaries. This tessellation method has already been developed by the current authors [5]. As the next step, equations of motion for a deformable C.V. need to be coupled with the tessellation method to calculate the instantaneous pressures in a two-phase flow field, with a moving interface, which will be the ultimate goal of the current study.

The conservation equations of volume, mass, and momentum, governing an isotropic fluid flow, embedded in a control volume (C.V.) with deformable surface boundaries are formulated as [6]:

$$
\begin{aligned}
& \frac{d V}{d t}=\int_{S(t)} \mathbf{u}_{\mathrm{S}} \cdot \mathbf{n} d A, \\
& \frac{d(\rho V)}{d t}+\int_{S(t)} \rho\left(\mathbf{u}-\mathbf{u}_{\mathrm{S}}\right) \cdot \mathbf{n} d A=0, \\
& \frac{d(\rho \mathbf{u} V)}{d t}+\int_{S(t)} \rho \mathbf{u}\left(\mathbf{u}-\mathbf{u}_{\mathrm{S}}\right) \cdot \mathbf{n} d A=\int_{V(t)}(\rho \mathbf{g}-\nabla p+\sigma \kappa \mathbf{n}) d V+\int_{V(t)} \mu\left(\nabla \mathbf{u}+\nabla \mathbf{u}^{\mathrm{T}}\right) \cdot \mathbf{n} d A,
\end{aligned}
$$

where $S(t)$ and $V(t)$ are the time-dependent control surface (C.S.) and C.V. of a cell. Here, $\mathbf{n}$ is normal to the surface area of $A$, pointing outward. The fluid and the control surface velocities are depicted by $\mathbf{u}$ and $\mathbf{u}_{\mathrm{s}}$, respectively and $\rho$ and $\mu$ are the density and dynamic viscosity of the fluid. $p$ is pressure, and $\mathbf{g}$ is the gravitational acceleration vector. The surface tension coefficient is shown by $\sigma$, and the curvature of the interface between two-phases is defined as $\kappa=\nabla \cdot \mathbf{n}$. Here, the surface tension force was modeled as a body force [7]. Equation (1) acts as a geometrical restrain, describing the changing rate of the volume as a result of moving surface boundaries of the C.V. [6]. The set of equations (2)-(3) can be discretized using high-order schemes, which need to satisfy equation (1) for a dynamic mesh of that time step.

Different scenarios of a gas-liquid two-phase flow, with the gas phase being the ambient air and the liquid phase a mixture of pure glycerol and distilled water were generated using an experimental setup based on the particle shadow velocimetry (PSV) [8]. Two versions of capillaries were manufactured using stereolithography apparatus (SLA) additive manufacturing technique: a straight square channel with a cross-sectional area of $3 \times 3 \mathrm{~mm}^{2}$, and a sinusoidal channel with a throat size of $0.5 \mathrm{~mm}$, depth of $1 \mathrm{~mm}$, amplitude of $1.25 \mathrm{~mm}$ and wavelength of $12 \mathrm{~mm}$. The straight and sinusoidal channels were designed to generate a single moving non-deformable bubble, and multiple moving deformable bubbles, respectively.

As shown in Figure 1, from PTV (or PIV) a dense velocity field can be calculated in the liquid phase, which means $\mathbf{u}$ in equations (1)-(3) becomes a known parameter. Information of the fluid volume and the velocity 
of the surface boundaries at each time can be extracted by an accurate image-processing algorithm. Identification of the temporal evolution of the two-phase interface makes it feasible to calculate the curvature $\kappa(t)$ in equation (3). With $V, \mathbf{u}, \mathbf{u}_{\mathrm{s}}$ and $\kappa$ known at each time, the set of equations (1)-(3) simplifies to an equation with the pressure as the only unknown, which, with a proper definition of the boundary conditions, and a suitable iterative method is solvable. To do this, the first step is to interconnect the sparse PTV velocity vectors in the flow domain using a high-quality mesh grid, which needs to dynamically adjust to the deformable and moving boundaries of the flow field. This part of the algorithm has been successfully developed and validated, for which the details are given in Azadi et al. [5]. Figure 1 represents the sample tessellation results for two different snapshots of the gas-liquid flow field in the sinusoidal channel. Here, the outline of the bubble moves and deforms with time, which imposes moving boundaries to the control volume embedding the liquid flow, hence demanding a dynamic mesh to tessellate and to solve for pressure.

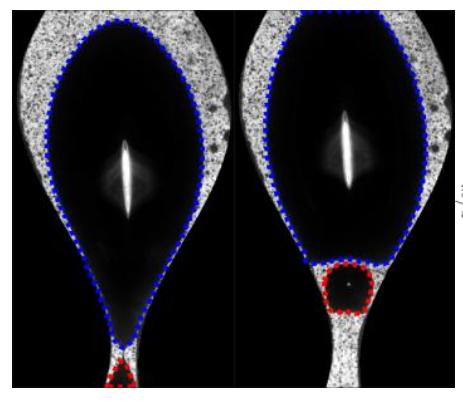

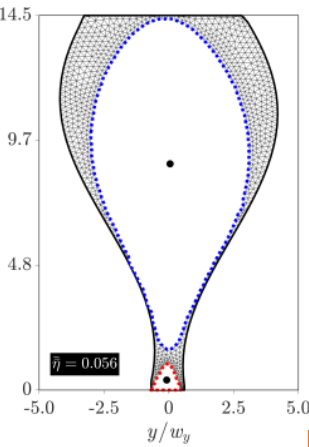

Image processing [5] \& PTV

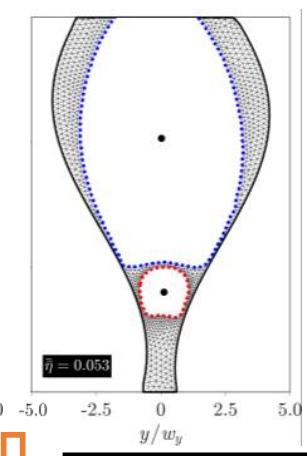

Dynamic meshing \& tessellation [5] $S_{\text {ext }}, S_{\text {int }}(t), \mathbf{u}_{\mathrm{s}}(t)$ $A(\mathbf{x}, t), V(\mathbf{x}, t), \mathbf{n}(\mathbf{x}, t), \kappa\left(S_{\text {int }}, \mathbf{n}_{\text {int }}, t\right)$
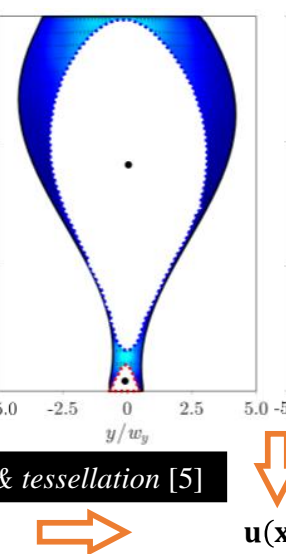

$\mathbf{u}(\mathbf{x}, t)$

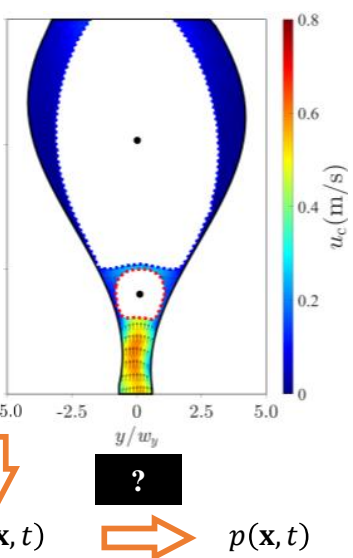

Figure 1: Snapshots of a gas-liquid flow field, with moving and deformable interfaces in a sinusoidal capillary for two different instants. Here, (a) shows the raw images of the seeded liquid field and the outlines of the bubbles. The highquality mesh domains shown in (b) are the results of applying the tessellation method of Azadi et al. [5]. (c) The PTV velocity field interpolated on the mesh domains shown in (b), with interpolated uniform vectors drawn on top of them.

The resultant dynamic mesh and the interconnected PTV velocity field, shown in Figure 1, along with the calculated interface curvature from image processing, can be introduced to each cell in the domain, using equation (3), to calculate for the instantons pressure field. Here, the accuracy of the PTV data, the selection of proper discretization schemes, the tessellation and curvature calculation methods used, all impact the final calculated pressure. For the full paper, details of the developed algorithms will be presented, and the applicability of the method will be demonstrated through applying it to several analytical problems and experimental data for flow scenarios in the straight and sinusoidal channels.

\section{References}

[1] Raffel M, Willert C E, Scarano F, Kähler C J, Wereley S T and Kompenhans J 2018 Particle Image Velocimetry (Cham: Springer International Publishing)

[2] Schanz D, Gesemann S and Schröder A 2016 Shake-The-Box: Lagrangian particle tracking at high particle image densities Exp. Fluids $\mathbf{5 7} 70$

[3] Auteri F, Carini M, Zagaglia D, Montagnani D, Gibertini G, Merz C B and Zanotti A 2015 A novel approach for reconstructing pressure from PIV velocity measurements Exp. Fluids $\mathbf{5 6} 45$

[4] Schneiders J F G, Caridi G C A, Sciacchitano A and Scarano F 2016 Large-scale volumetric pressure from tomographic PTV with HFSB tracers Exp. Fluids $\mathbf{5 7} 164$

[5] Azadi R, Wong J and Nobes D S 2021 Determination of Fluid Flow Adjacent to a Gas/Liquid Interface Using Particle Tracking Velocimetry (PTV) And a High-Quality Tessellation Approach Exp. Fluids (In pub)

[6] Perot B and Nallapati R 2003 A moving unstructured staggered mesh method for the simulation of incompressible free-surface flows J. Comput. Phys. 184 192-214

[7] Brackbill J ., Kothe D . and Zemach C 1992 A continuum method for modeling surface tension J. Comput. Phys. $100335-54$

[8] Estevadeordal J and Goss L 2005 PIV with LED: Particle Shadow Velocimetry (PSV) Technique 43rd AIAA Aerospace Sciences Meeting and Exhibit (Reston, Virigina: American Institute of Aeronautics and Astronautics) 